\title{
Right ventricular reserve in a piglet model of chronic pulmonary hypertension
}

\author{
Julien Guihaire1, François Haddad², Pierre-Emmanuel Noly , David Boulate \\ Benoit Decante ${ }^{1}$, Philippe Dartevelle ${ }^{1}$, Marc Humbert ${ }^{3}$, Jean-Philippe Verhoye ${ }^{4}$, \\ Olaf Mercier ${ }^{1}$ and Elie Fadel ${ }^{1}$
}

Affiliations: 'Laboratory of Surgical Research and INSERM U999, University of Paris-Sud, Marie Lannelongue Hospital, Le Plessis Robinson, France. ${ }^{2}$ Division of Cardiovascular Medicine, Stanford University, Palo Alto, CA, USA. ${ }^{3}$ University of Paris-Sud, Faculté de médecine, Kremlin-Bicêtre, AP-HP, Service de Pneumologie et Réanimation Respiratoire, Hôpital Bicêtre, Le Kremlin-Bicêtre, INSERM U999, Marie Lannelongue Hospital, Le Plessis-Robinson, France. ${ }^{4}$ Dept of Thoracic and Cardiovascular Surgery, University Hospital of Rennes, Rennes, France.

Correspondence: Julien Guihaire, Laboratory of Surgical Research and Inserm U999, University of Paris-Sud, Marie Lannelongue Hospital, 133 avenue de la Resistance, 92350 Le Plessis Robinson, France.

E-mail: julien.guihairedechu-rennes.fr

ABSTRACT Right ventricular (RV) response to exercise or pharmacological stress is not well documented in pulmonary hypertension $(\mathrm{PH})$. We investigated the relationship between RV reserve and ventricular-arterial coupling.

Surgical ligation of the left pulmonary artery was performed in 13 Large White piglets ( $\mathrm{PH}$ group), thereafter weekly embolisations of the right lower lobe were performed for 5 weeks. A control group of six piglets underwent sham procedures. Right heart catheterisation and echocardiography were performed at week 6. Pressure-volume loops were recorded before and after dobutamine infusion.

Induction of experimental $\mathrm{PH}$ resulted in a higher mean $\pm \mathrm{SD}$ pulmonary artery pressure ( $34 \pm 9$ versus $14 \pm 2 \mathrm{mmHg} ; \mathrm{p}<0.01)$ and in a lower ventricular-arterial coupling efficiency $(0.66 \pm 0.18$ versus $1.24 \pm 0.17$; $\mathrm{p}<0.01)$ compared with controls at 6 weeks. Dobutamine-induced relative changes in RV stroke volume index (SVI) and end-systolic elastance were lower in the $\mathrm{PH}$ group (mean \pm SD $47 \pm 5 \%$ versus $20 \pm 5 \%$, $\mathrm{p}<0.01$, and $81 \pm 37 \%$ versus $32 \pm 14 \%, \mathrm{p}<0.01$, respectively). Change in SVI was strongly associated with resting ventricular-arterial coupling $\left(\mathrm{R}^{2}=0.74 ; \mathrm{p}<0.01\right)$.

RV reserve was associated with ventricular-arterial coupling in a porcine model of chronic pressure overload.

@ERSpublications

Dobutamine testing in right ventricular pressure overload: a potentially useful method to assess contractile reserve http://ow.ly/E9NMK

For editorial comments see Eur Respir J 2015; 45: 604-607 [DOI: 10.1183/09031936.00233614].

This article has supplementary material available from erj.ersjournals.com

Received: May 012014 | Accepted after revision: Nov 062014 | First published online: Dec 102014

Support statement: This work was supported by the "Association chirurgicale pour le développement et l'amélioration des techniques de dépistage et de traitement des maladies cardio-vasculaires" (ADETEC, Suresnes, France). The Vivid E9 cardiac ultrasound system (General Electric Medical System) was financed by a grant from the "Cardio-vasculaire-Obésité Domaine D’Intérêt Majeur" (CODDIM cod100158, Région Ile-de-France, France).

Conflict of interest: Disclosures can be found alongside the online version of this article at erj.ersjournals.com 


\section{Introduction}

Survival in patients with pulmonary hypertension $(\mathrm{PH})$ is closely related to right ventricular (RV) function [1-3]. In haemodynamics-based studies, low cardiac index (CI) and increased right atrial pressure (RAP) at rest are strong predictors of outcome [4]. While the majority of studies have focused on evaluating RV function at rest, in recent years there has been a renewed interest in evaluating the dynamic response of the right ventricle to either exercise or pharmacological stimulation [5-7]. Nootens et al. [8] were the first to describe the significant decrease in contractile reserve in patients with PH. In their study, RV ejection fraction failed to increase and actually decreased with exercise in the majority of patients with moderate-to-severe pulmonary vascular disease.

Currently, the physiological correlates of contractile reserve in the chronic RV pressure overload state are not well understood. In this experimental study, we sought to determine whether contractile reserve in $\mathrm{PH}$ is strongly associated with ventricular-arterial coupling. Ventricular-arterial coupling is a measure that relates ventricular contractility to ventricular afterload and is currently the reference metric for functional ventricular adaptation [9, 10]. For this study, we hypothesised that RV contractile reserve in the chronic pressure overload state would represent a good surrogate marker for ventricular-arterial coupling. We also sought to determine whether contractile reserve is associated with $\beta$-myosin heavy chain ( $\beta$-MHC) expression.

\section{Materials and methods}

\section{Experimental model of chronic PH}

After premedication with ketamine hydrochloride $15 \mathrm{mg} \cdot \mathrm{kg}^{-1}$ intramuscularly, general anaesthesia was induced with propofol $1 \%$ and maintained using continuous isoflurane inhalation for all procedures (surgery, right heart catheterisation, conductance measurements and echocardiography). Pancuronium bromide $2 \mathrm{mg}$ was administrated intravenously for muscle relaxation. Mechanical ventilation via endotracheal intubation was performed at a rate of 10 breaths per minute, with a tidal volume of $15-20 \mathrm{~mL} \cdot \mathrm{kg}^{-1}$ and a pulmonary end-expiratory pressure of $5 \mathrm{cmH}_{2} \mathrm{O}$ (Servo 900; Siemens-Elema $\mathrm{AB}$, Solna, Sweden). Inspired oxygen fraction was adjusted to obtain stable peripheral oxygen saturation up to 95\% during all procedures. 13 2-month-old Large White piglets underwent a ligation of the left pulmonary artery through midline sternotomy (PH group). Thereafter, percutaneous puncture of the brachiocephalic vein was carried out weekly to perform pulmonary embolisations once per week for 5 weeks using a 4-Fr lumen catheter. A soft tissue adhesive, N-butyl-2-cyanoacrylate (Histoacryl; B-Braun, Tuttlingen, Germany), was then injected under fluoroscopic guidance to progressively occlude the segmental arteries of the right lower lobe. As previously reported, the animals developed $\mathrm{PH}$ associated with RV remodelling and dysfunction after 5 weeks [11]. Sham surgical procedures were performed in six piglets (Sham group). The pericardium was kept open in both groups. At week 6, $\mathrm{PH}$ animals were randomly allocated to either a surgical reperfusion of the left lung using a short bypass between the pulmonary artery trunk and the left pulmonary artery (reperfusion group, $n=6$ ), or no treatment (HYPPE group, $n=7$ ). The research protocol was approved by the Institutional Committee on Animal Welfare. All animals received humane care in compliance with the "Principles of Laboratory Animal Care" formulated by the National Society for Medical Research and the "Guide for the Care and Use of Laboratory Animals" prepared by the Institute of Laboratory Animal Resources and published by the National Institutes of Health (NIH Publication No. 86-23, revised 1996). The study was performed in the Laboratory of Surgical Research of Marie Lannelongue Hospital, University of Paris-Sud, Le Plessis Robinson, France.

\section{Right heart catheterisation}

Pulmonary haemodynamics including heart rate, RAP, systolic and mean pulmonary artery pressure (sPAP and mPAP) and cardiac output were derived by thermodilution under general anaesthesia at week 6 in all animals using a 7-Fr catheter (Edwards LifeSciences, Irvine, CA, USA). Total pulmonary resistance (TPR) was expressed in $\mathrm{dyn} \cdot \mathrm{s}^{-1} \cdot \mathrm{cm}^{-5}$ and calculated as follows: TPR $=((\mathrm{mPAP}) \times 80) /$ cardiac output. Cardiac output was indexed to the body surface area (BSA) to define CI following the equation of KelLey et al. [12] for piglets: BSA $\left(\mathrm{cm}^{2}\right)=734 \times$ weight $^{0.656}$. Cardiac power index was calculated as follows: $\mathrm{mPAP} \times \mathrm{CI}$.

\section{Conductance measurements}

Synchronous pressure and volume signals were recorded at weeks 6 and 12 using a 4-Fr conductance-catheter (CD Leycom, Zoetermeer, the Netherlands) inserted in the right ventricle as previously reported [11, 13]. Another pressure sensitive catheter (Pulsion Medical Systems, Munich, Germany) was inserted in the right femoral artery to continuously monitor mean systemic blood pressure. Baseline measurements for stroke volume, maximal $(\mathrm{dP} / \mathrm{dtmax})$ and minimal rates of rise of RV pressure, relaxation time constant (tau) and RV stroke work were recorded. Stroke volume was indexed to BSA (SVI). An 8-Fr balloon-tipped catheter was introduced through the right femoral vein and positioned under fluoroscopic guidance in the inferior vena cava, just below the right atrial cavity $(20-\mathrm{mm}$ occlusion 
balloon catheter; Boston Scientific/Medi-Tech, Watertown, MA, USA). It was inflated to transiently reduce RV inflow and assess end-systolic pressure-volume relationships. End-systolic elastance of the right ventricle (Ees) was defined by the slope of the end-systolic pressure-volume relationship. Pulmonary arterial elastance $(\mathrm{Ea})$ was calculated as the ratio of RV end-systolic pressure to stroke volume. The Ees/Ea ratio determined the efficiency of RV-pulmonary arterial (RV-PA) coupling. Measurements (heart rate, stroke volume, $\mathrm{dP} / \mathrm{dtmax}$ and Ees) were repeated after $3 \mathrm{~min}$ of low-dose dobutamine infusion $\left(5 \mu \mathrm{g} \cdot \mathrm{kg}^{-1} \cdot \mathrm{min}^{-1}\right)$. Different metrics of RV contractile reserve were reported, reflecting relative changes in indices of systolic function: 1) (SVI after stress - SVI at rest)/SVI at rest; 2) (dP/dtmax after stress - dP/dtmax at rest)/dP/dtmax at rest; and 3) (Ees after stress - Ees at rest)/Ees at rest. We also defined three categories with respect to the degree of RV-PA coupling: moderate-to-severe $(\mathrm{Ees} / \mathrm{Ea}<0.7)$, mild $(0.7<\mathrm{Ees} / \mathrm{Ea}<1)$ and normal (Ees/Ea).

\section{Echography of the right ventricle}

Echocardiography (Vivid E9; GE Medical Systems, Milwaukee, WI, USA) was performed at week 6 under general anaesthesia for measurement of the maximal transverse RV end-diastolic diameter (RVEDD) on a 4-chamber view (B-mode), as well as the RV end-diastolic area indexed to BSA (RVEDAI). The RV fractional area change (RVFAC) was defined as the percentage change in area between end-diastole and end-systole (two-dimensional quantification). The RV myocardial performance index was calculated as the ratio of isovolumic contraction and relaxation time divided by contraction time (pulse doppler of the pulmonary and tricuspid valves). The tricuspid annular plane systolic excursion (TAPSE) was measured using M-mode as the maximal excursion of the lateral part of the tricuspid annulus.

\section{Reverse transcriptase PCR assay}

Animals were euthanised at week 12 for procurement of the right ventricle. Total RNA was prepared and quantified according to a method we previously described [14]. Reverse transcriptase (RT)-PCR was performed for brain natriuretic peptide (BNP) and $\beta$-MHC with pig-specific primers (\#Ss03392423_m1 and \#Ss03392411_m1, respectively; Applied Biosystems, Villebon sur Yvette, France). Tissue expression of $\beta-\mathrm{MHC}$ and BNP was normalised to that of the $18 \mathrm{~S}$ gene in each sample.

\section{Statistical analysis}

Continuous variables are presented as mean \pm SD. Non-normally distributed variables were log transformed before analysis. Differences between study groups were detected using one-way ANOVA. Within groups, changes after pharmacological stimulation were evaluated using paired t-tests. Relationships between different modalities of RV contractility assessment and RV-PA coupling or with noninvasive indices of RV function were approached using linear regression. $p<0.05$ was considered significant. All data were analysed using Prism statistical software (GraphPad, La Jolla, CA, USA).

\section{Results}

Haemodynamics and RV remodelling after induction of experimental $\mathrm{PH}$ Characteristics of the study groups at week 6 are listed in table 1.

Left pulmonary artery ligation combined with embolisation of the segmental arteries of the right lower lobe for 5 weeks resulted in significant changes in pulmonary haemodynamics as illustrated by the higher RAP, mPAP and TPR among PH animals. Heart rate and CI were not different between the PH and sham groups, while cardiac power index and RV stroke work were higher at 6 weeks after induction of experimental PH. Chronic pressure overload was associated with RV enlargement as shown by the higher values of RVEDD and RVEDAI among PH animals. Moreover, RV systolic dysfunction was observed in $\mathrm{PH}$ animals as illustrated by the lower RVFAC and TAPSE compared with controls. The RVFAC/sPAP and TAPSE/sPAP ratios were significantly lower in the PH group. Similarly, we observed a linear relationship between RVFAC and sPAP, and between TAPSE and sPAP at rest (fig. S1). Resting parameters of RV systolic function were weakly associated with Ees/Ea (TAPSE: $\mathrm{R}^{2}=0.41, \mathrm{p}<0.01$; SVI: $\mathrm{R}^{2}=0.22, \mathrm{p}=0.04$; dP/dtmax: $\mathrm{R}^{2}=0.39, \mathrm{p}<0.01$; Ees: $\left.\mathrm{R}^{2}=0.42, \mathrm{p}<0.01\right)$, except for $\operatorname{RVFAC}\left(\mathrm{R}^{2}=0.82 ; \mathrm{p}<0.01\right)$.

\section{$R V$ contractile reserve}

At rest, indices of RV contractility, including $\mathrm{dP} / \mathrm{dtmax}$ and Ees, were significantly higher in the $\mathrm{PH}$ group. Impairment of ventricular-arterial coupling was, however, observed in this group; since the increased Ees did not compensate for the increase in Ea. Changes in RV haemodynamics after pharmacological stress at week 6 are shown in figure 1. Dobutamine infusion $\left(5 \mu \mathrm{g} \cdot \mathrm{kg}^{-1} \cdot \mathrm{min}^{-1}\right)$ resulted in a significant increase in heart rate $\left(101 \pm 16\right.$ versus $131 \pm 17$ beats $\left.\cdot \mathrm{min}^{-1} ; \mathrm{p}<0.01\right)$, SVI $(41.5 \pm 6.6$ versus $50.1 \pm 7.8 \mathrm{~mL} ; \mathrm{p}<0.01)$ and $\mathrm{CI}(3.2 \pm 1.1$ versus $4.7 \pm 1.3 \mathrm{~L} \cdot \mathrm{min}^{-1} \cdot \mathrm{m}^{-2}$; $\left.\mathrm{p}<0.01\right)$ in the $\mathrm{PH}$ group. Similarly, pharmacological stress was associated with an improvement in RV contractility as measured by Ees $\left(0.53 \pm 0.08\right.$ versus $\left.0.70 \pm 0.12 \mathrm{mmHg} \cdot \mathrm{mL}^{-1} ; \mathrm{p}<0.01\right)$ and 
TABLE 1 Resting haemodynamics, right ventricular (RV) remodelling and function at 6 weeks

\begin{tabular}{|c|c|c|c|}
\hline & $\mathrm{PH}$ group & Sham group & p-value \\
\hline Subjects $n$ & 13 & 6 & \\
\hline Body weight kg & $31.9 \pm 5.6$ & $30.6 \pm 2.2$ & 0.59 \\
\hline Mean blood pressure $\mathrm{mmHg}$ & $63 \pm 13$ & $70 \pm 11$ & 0.25 \\
\hline Heart rate beats. $\min ^{-1}$ & $100 \pm 16$ & $101 \pm 13$ & 0.93 \\
\hline \multicolumn{4}{|l|}{ Right heart catheterisation } \\
\hline RAP $\mathrm{mmHg}$ & $8 \pm 2$ & $5 \pm 2$ & 0.01 \\
\hline RV end-diastolic pressure & $13 \pm 5$ & $5 \pm 3$ & $<0.01$ \\
\hline Mean PAP $\mathrm{mmHg}$ & $34 \pm 9$ & $14 \pm 2$ & $<0.01$ \\
\hline Cardiac index $\mathrm{L} \cdot \mathrm{min}^{-1} \cdot \mathrm{m}^{-2}$ & $3.2 \pm 1.0$ & $3.2 \pm 0.2$ & 0.93 \\
\hline TPR dyn $\cdot \mathrm{s} \cdot \mathrm{cm}^{-5}$ & $993 \pm 572$ & $349 \pm 61$ & 0.01 \\
\hline Cardiac power index $\mathrm{W} \cdot \mathrm{m}^{-2}$ & $108 \pm 42$ & $45 \pm 6$ & $<0.01$ \\
\hline \multicolumn{4}{|l|}{ Conductance measurements } \\
\hline $\mathrm{SVI} \mathrm{mL} \cdot \mathrm{m}^{-2}$ & $41 \pm 7$ & $44 \pm 5$ & 0.49 \\
\hline $\mathrm{RV}$ stroke work $\mathrm{mmHg} \cdot \mathrm{mL}^{-1}$ & $1853 \pm 851$ & $881 \pm 265$ & 0.03 \\
\hline $\mathrm{dP} / \mathrm{dtmax} \mathrm{mmHg} \cdot \mathrm{s}^{-1}$ & $540 \pm 141$ & $306 \pm 72$ & $<0.01$ \\
\hline $\mathrm{dP} / \mathrm{dtmin} \mathrm{mmHg} \cdot \mathrm{s}^{-1}$ & $-738 \pm 299$ & $-244 \pm 65$ & $<0.01$ \\
\hline Ees $\mathrm{mmHg} \cdot \mathrm{mL}^{-1}$ & $0.54 \pm 0.09$ & $0.37 \pm 0.05$ & $<0.01$ \\
\hline Ea $\mathrm{mmHg} \cdot \mathrm{mL}^{-1}$ & $0.88 \pm 0.35$ & $0.32 \pm 0.06$ & $<0.01$ \\
\hline $\mathrm{Ees} / \mathrm{Ea}$ & $0.66 \pm 0.18$ & $1.24 \pm 0.17$ & $<0.01$ \\
\hline Tau ms & $34 \pm 4$ & $18 \pm 7$ & $<0.01$ \\
\hline \multicolumn{4}{|l|}{ Echocardiography } \\
\hline LVEF \% & $51 \pm 2$ & $62 \pm 3$ & $<0.01$ \\
\hline $\mathrm{RV}$ end-diastolic diameter $\mathrm{cm}$ & $2.1 \pm 0.6$ & $1.4 \pm 0.3$ & 0.02 \\
\hline $\mathrm{RV}$ end-diastolic area index $\mathrm{cm}^{2} \cdot \mathrm{m}^{-2}$ & $11.0 \pm 2.4$ & $4.6 \pm 0.6$ & $<0.01$ \\
\hline RVFAC & $0.25 \pm 0.04$ & $0.51 \pm 0.08$ & $<0.01$ \\
\hline RV myocardial performance index & $0.62 \pm 0.17$ & $0.30 \pm 0.04$ & $<0.01$ \\
\hline TAPSE cm & $1.4 \pm 0.4$ & $2.0 \pm 0.2$ & $<0.01$ \\
\hline $\mathrm{RVFAC} / \mathrm{sPAP} \mathrm{mm} \cdot \mathrm{mmHg}^{-1}$ & $0.63 \pm 0.05$ & $3.05 \pm 0.32$ & $<0.01$ \\
\hline TAPSE/sPAP $\mathrm{mm} \cdot \mathrm{mmHg}^{-1}$ & $0.34 \pm 0.09$ & $1.07 \pm 0.15$ & $<0.01$ \\
\hline
\end{tabular}

Data are presented as mean $\pm S D$, unless otherwise stated. Statistical significance was set at $p<0.05$. $P H$ : pulmonary hypertension; RAP: right atrial pressure; PAP: pulmonary artery pressure; TPR: total pulmonary resistance; SVI: stroke volume index; $d P / d t m a x:$ maximum rate of rise of RV pressure; $d P / d t m i n:$ minimum rate of rise of RV pressure; Ees: end-systolic elastance; Ea: arterial elastance; LVEF: left ventricular ejection fraction; RVFAC: RV fractional area change; TAPSE: tricuspid annular plane systolic excursion; sPAP: systolic PAP.

higher $\mathrm{dP} / \mathrm{dtmax}\left(540 \pm 141\right.$ versus $1079 \pm 353 \mathrm{mmHg} \cdot \mathrm{s}^{-1}$; $\left.\mathrm{p}<0.01\right)$ compared with measurements at rest. This increase in Ees was, however, insufficient to improve RV-PA coupling in $\mathrm{PH}$ animals $(0.66 \pm 0.18$ versus $0.55 \pm 0.21 ; \mathrm{p}=0.14$ ), while Ees/Ea was higher in sham animals after dobutamine infusion at week 6 $(1.23 \pm 0.17$ versus $2.14 \pm 0.55 ; \mathrm{p}=0.01)$.

Relationships between indices of RV contractile reserve, RV functional indices and ventriculararterial coupling

Pharmacological stress resulted in a marked increase in SVI, dP/dtmax and Ees in both groups. The relative changes during dobutamine stimulation were, however, higher in controls compared with $\mathrm{PH}$ animals (SVI: $47 \pm 5 \%$ versus $20 \pm 5 \%, \mathrm{p}<0.01 ; \mathrm{dP} / \mathrm{dtmax}$ : $214 \pm 55 \%$ versus $95 \pm 51 \%, \mathrm{p}<0.01$; Ees: $81 \pm 37 \%$ versus $32 \pm 14 \%, \mathrm{p}<0.01$ ) (fig. 2).

The relationships between indices of RV contractile reserve and traditional indices of RV function at rest are listed in table 2. The RVFAC at rest was more strongly associated with SVI reserve, Ees reserve and $\mathrm{dP} / \mathrm{dtmax}$ reserve through linear relationships, than the TAPSE and RVMPI. The TAPSE was not associated with relative changes in Ees after dobutamine infusion $\left(\mathrm{R}^{2}=0.172 ; \mathrm{p}=0.078\right)$.

The lower the Ees/Ea ratio was, the lower the SVI was in response to dobutamine (fig. 3a). The relationships between indices of contractile reserve and RV-PA coupling at rest (Ees/Ea) were better approximated by a linear approach (fig. 3b-d). Impairment of RV-PA coupling $(\mathrm{Ees} / \mathrm{Ea}<1$ ) was associated with lower changes in SVI and dP/dtmax after dobutamine stimulation. Resting Ees/Ea was more strongly associated with relative changes in SVI $\left(R^{2}=0.74 ; \mathrm{p}<0.01\right)$ and in $\mathrm{dP} / \mathrm{dtmax}\left(\mathrm{R}^{2}=0.70 ; \mathrm{p}<0.01\right)$, than with relative change in Ees $\left(\mathrm{R}^{2}=0.36 ; \mathrm{p}<0.01\right)$. 

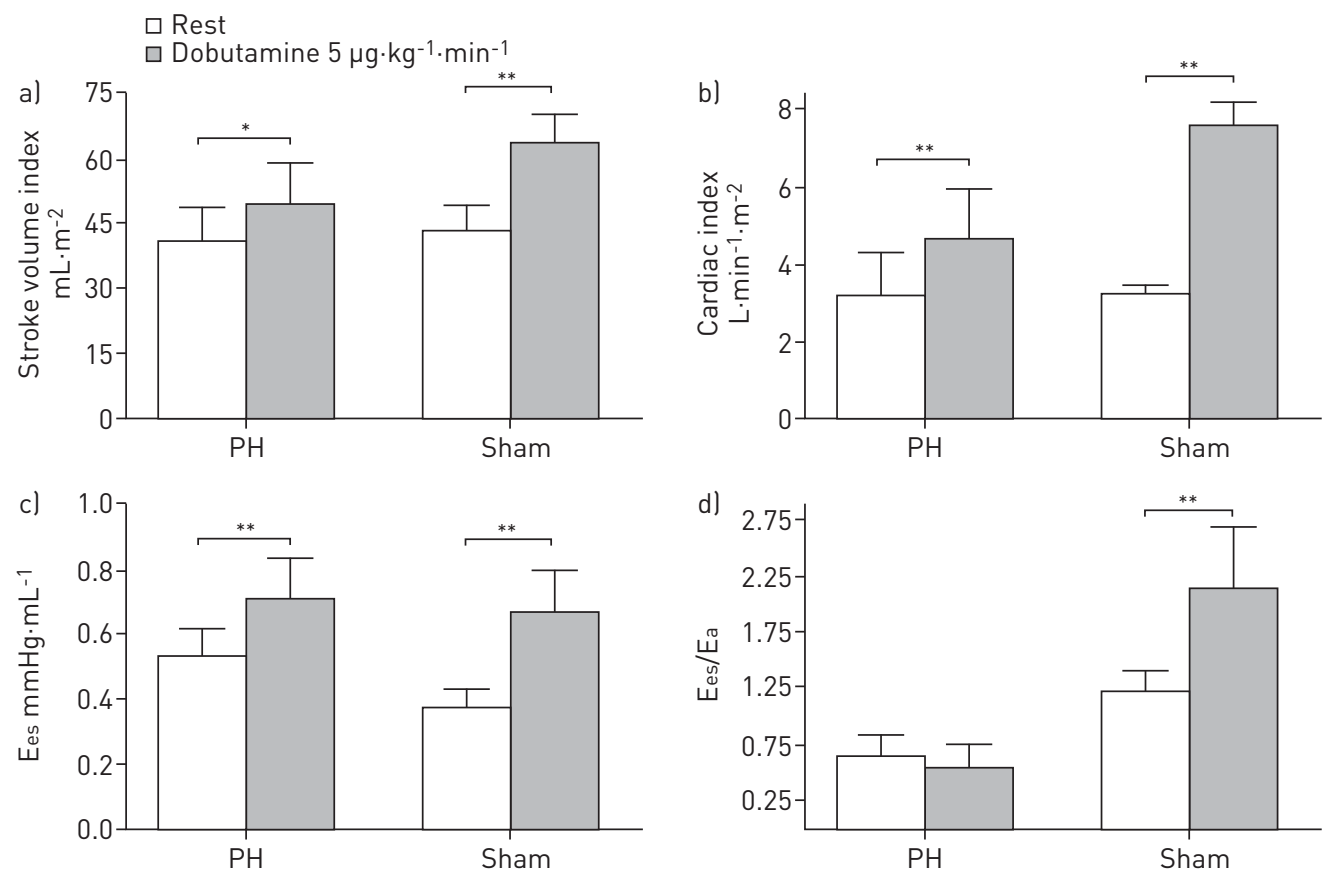

FIGURE 1 Changes in right ventricular haemodynamics during a pharmacological stress test. a) Stroke volume index, b) cardiac index, c) end-systolic elastance (Ees) and d) Ees/pulmonary arterial elastance (Ea). Pulmonary hypertension $(\mathrm{PH})$ group $\mathrm{n}=13$; sham group $\mathrm{n}=6{ }^{*}: \mathrm{p}<0.05{ }^{* *}$ : $\mathrm{p}<0.01$ for comparison between resting and exercise variables in each group.
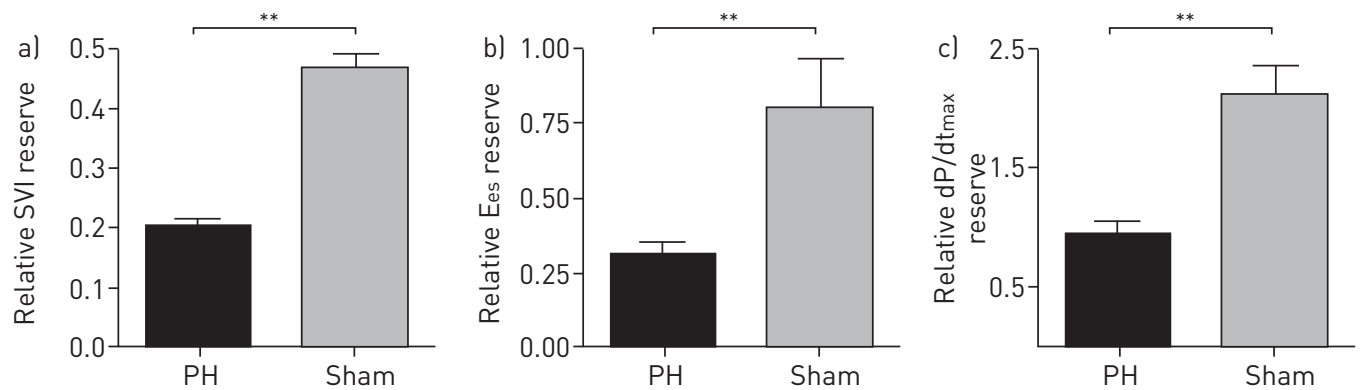

FIGURE 2 Comparison of different metric of right ventricular contractile reserve between the pulmonary hypertension $(\mathrm{PH})$ and sham groups. a) Relative stroke volume index (SVI), defined as relative SVI= (maximum SVI-SVI at rest)/ SVI at rest. b) Relative end-systolic elastance $(\mathrm{Ees})$, defined as relative Ees $=($ maximum Ees - Ees at rest $) /$ Ees at rest. c) Relative maximum rate of rise of right ventricular pressure $(\mathrm{dP} / \mathrm{dtmax})$, defined as relative $\mathrm{dP} / \mathrm{dtmax}=($ maximum $\mathrm{dP} / \mathrm{dt}$ $-\mathrm{dP} / \mathrm{dt}$ at rest $) / \mathrm{dP} / \mathrm{dt}$ at rest. $\mathrm{PH}$ group $\mathrm{n}=13$; sham group $\mathrm{n}=6{ }^{* *}: \mathrm{p}<0.01$ for between group comparisons.

TABLE 2 Relationships between noninvasive indices of right ventricular (RV) function at rest and new indices of RV contractile reserve at 6 weeks

\begin{tabular}{|c|c|c|c|c|c|c|}
\hline \multirow[t]{2}{*}{ Indices of RV function } & \multicolumn{2}{|c|}{ SVI reserve } & \multicolumn{2}{|c|}{ Ees reserve } & \multicolumn{2}{|c|}{$\mathrm{dP} / \mathrm{dtmax}$ reserve } \\
\hline & $\mathbf{R}^{2}$ & $\overline{p \text {-value }}$ & $\mathbf{R}^{2}$ & p-value & $\mathbf{R}^{2}$ & $\overline{p \text {-value }}$ \\
\hline TAPSE & 0.462 & 0.001 & 0.172 & 0.078 & 0.296 & 0.018 \\
\hline RVMPI & 0.560 & $<0.001$ & 0.313 & 0.013 & 0.462 & 0.001 \\
\hline RVFAC & 0.699 & $<0.001$ & 0.316 & 0.012 & 0.573 & $<0.001$ \\
\hline
\end{tabular}

Associations were approximated using a linear approach. Statistical significance was set at $p<0.05$. SVI: stroke volume index; Ees: end-systolic elastance; dP/dtmax: maximum rate of rise of RV pressure; TAPSE: tricuspid annular plane systolic excursion; RVMPI: RV myocardial performance index; RVFAC: RV fractional area change. 

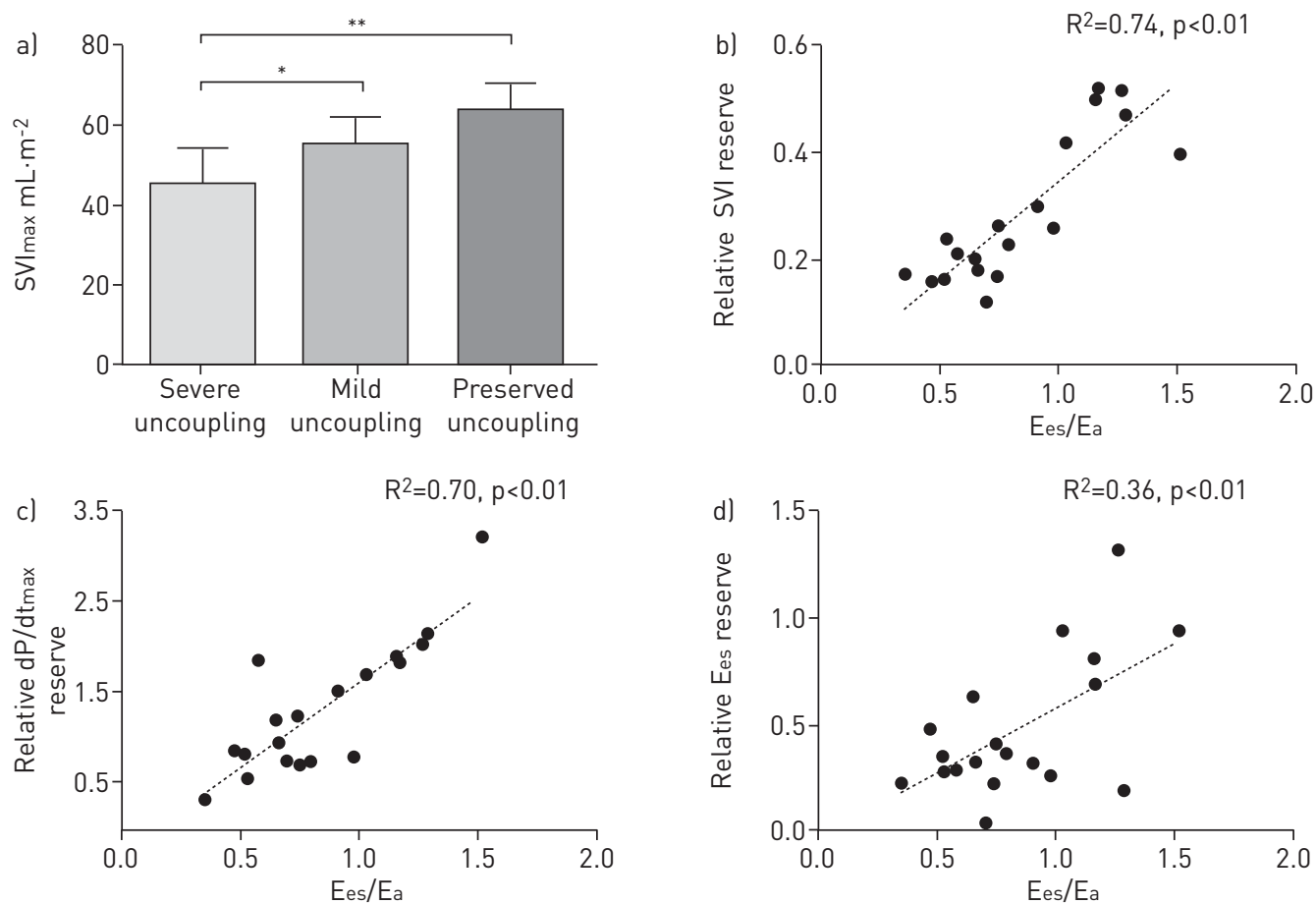

FIGURE 3 Relationships between indices of right ventricular contractile reserve and resting ventricular-arterial coupling $(\mathrm{Ees} / \mathrm{Ea})$. a) The more ventricular-arterial coupling impairment the lower the stroke volume index response to dobutamine stimulation (SVImax) was. Severe uncoupling was defined as Ees/Ea $<0.7$; moderate uncoupling as $0.7<\mathrm{Ees}_{\mathrm{e}} / \mathrm{E}_{\mathrm{a}}<1.0$; and preserved coupling as $\mathrm{E}_{\mathrm{es}} / \mathrm{E}_{\mathrm{a}}>1$. Relationships between $\mathrm{Ees} / \mathrm{Ea}_{\mathrm{a}}$ and $\mathrm{b}$ ) relative SVI reserve, c) relative maximum rate of rise of right ventricular pressure $(\mathrm{dP} / \mathrm{dtmax})$ reserve, and $\mathrm{d})$ relative Ees reserve were best approximated using a linear approach. $n=19$. Ees: end-systolic elastance; Ea: pulmonary arterial elastance. ${ }^{*}: \mathrm{p}<0.05$; ${ }^{* *}$ : $p<0.01$ for comparison between groups.

\section{Relationship between gene expression and RV reserve}

At 12 weeks, $\mathrm{PH}$ animals who did not receive any treatment at 6 weeks had a higher level of gene expression for BNP and when compared with the reperfusion group (BNP: $2.55 \pm 1.49$ versus $1.80 \pm 1.47$, $\mathrm{p}<0.01 ; \beta$-MHC: $1.58 \pm 0.43$ versus $1.31 \pm 0.27, \mathrm{p}=0.02$ ) (fig. $4 \mathrm{a}$ and $\mathrm{b}$ ). Considering the relationships between gene expression and RV systolic reserve, as illustrated by SVI after dobutamine stimulation at 12 weeks, we observed a trend for an inverse relationship between BNP expression and RV reserve $\left(\mathrm{R}^{2}=0.343 ; \mathrm{p}=0.05\right)$. The relationship between $\beta$-MHC expression and maximum SVI was best estimated using a linear approach $\left(\mathrm{R}^{2}=0.65 ; \mathrm{p}<0.01\right)$. The strongest relationship between $\mathrm{RV}$ gene expression and $\mathrm{RV}$ reserve was observed with $\beta$-MHC (fig. $4 c$ and d).

\section{Discussion}

The main finding of our study is that in chronic RV pressure overload states, a decrease in RV contractile reserve is strongly associated with impairment in ventricular-arterial coupling. These physiological observations have clinical implications for both screening and management of patients with $\mathrm{PH}$.

Emphasis in $\mathrm{PH}$ has recently changed from focusing on either the pulmonary vasculature or the right ventricle to focusing on the cardiopulmonary unit. Insights from analysis of pressure-volume loops have demonstrated that a ventricle that adapts well to chronic $\mathrm{PH}$ is able to increase its contractility to match the increase in afterload [15]. Ventricular-arterial coupling is the metric of choice to analyse this matching between contractility and afterload. Consistent with previous clinical and experimental studies, we have found that altered ventricular-arterial coupling is common in the presence of chronic pressure overload states of the right ventricle [16].

Our study demonstrates that, in the setting of a chronic pressure overload state, RV contractile reserve may be a more sensitive marker of haemodynamic ventricular dysfunction. In fact, while SVI and CI were not significantly decreased at rest in the $\mathrm{PH}$ group, contractile reserve measured by peak or relative changes in SVI or CI were significantly impaired. The impairment in contractile reserve was also reflected by a lower relative increase in more specific indices of contractility, such as Ees and $\mathrm{dP} / \mathrm{dtmax}$, in the $\mathrm{PH}$ group. To the best of our knowledge, this is the first report of a strong relationship between contractile reserve and resting 

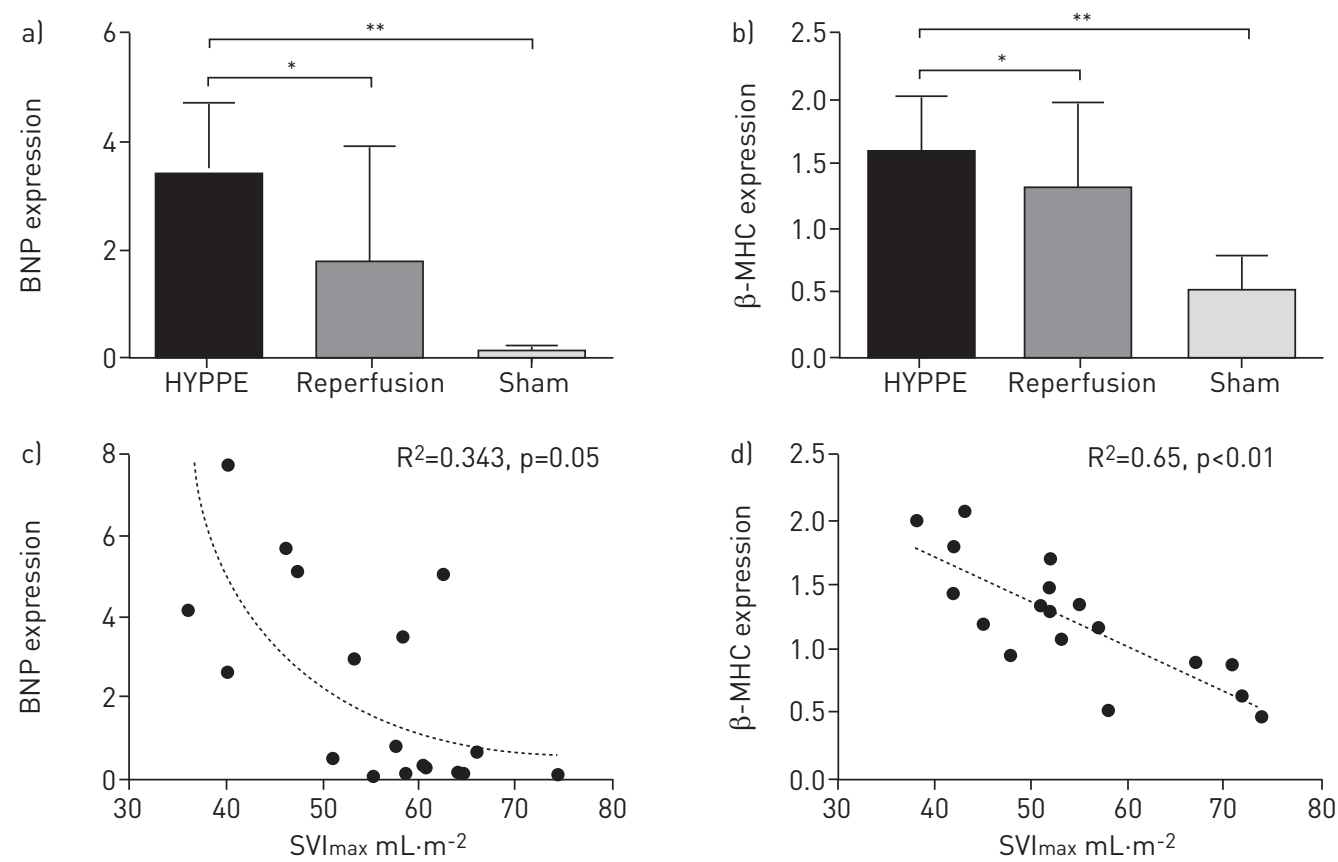

FIGURE 4 Molecular patterns of the pressure-overloaded right ventricle and relationships with right ventricular (RV) reserve. a) Brain natriuretic peptide (BNP) and b) $\beta$-myosin heavy chain ( $\beta$-MHC) are overexpressed at 12 weeks in HYPPE group $(n=7)$ compared with the reperfusion $(n=6)$ and sham groups $(n=6)$. RV gene expression of $c)$ BNP and d) $\beta$-MHC were inversely associated with stroke volume index measured after dobutamine stimulation (SVImax), through a non-linear and a linear relationship, respectively. ${ }^{*}: \mathrm{p}<0.05 ;{ }^{* *}: \mathrm{p}<0.01$ for comparison between resting and exercise variables in each group.

ventricular-arterial coupling in an experimental model of RV dysfunction. Several studies have previously demonstrated that functional changes during exercise are adaptive if the ventricle is energetically efficient, which is usually observed with an Ees/Ea ratio of 1-2 [17, 18]. Our findings are in accordance with studies showing that patients with $\mathrm{PH}$ have an impairment of power transfer and ventricular efficiency during exercise [11, 19]. Among functional indices at rest, RVFAC showed the best correlation to ventriculararterial coupling and contractile reserve, indicating that, if reliably measured, it could be a useful parameter for outcome or for screening. Our findings are in accordance with clinical results recently reported by RAINA et al. [20]. They showed that RVFAC better reflects RV systolic function than TAPSE when the pericardium is kept open. Practically, however, there may be more variability in the measure of RVFAC and recent outcome studies carried out by Fine et al. [21] suggest that RV longitudinal strain, which was not measured in the current study, is a stronger correlate of survival than RVFAC in prevalent cases of PH.

Although this was not specifically analysed in the study, several mechanisms can contribute to the decreased contractile reserve to dobutamine. First, the decrease in $\beta 1$-adrenergic receptor density in chronic RV pressure overload could explain, in part, the decrease in responsiveness to dobutamine. Secondly, the decrease in capillary density in the presence of a hypertrophied ventricle could contribute to perfusion abnormalities and inefficient myocardial oxygen utilisation leading to impaired contractile reserve [22-26]. Thirdly, a metabolic shift from fatty acid to glucose oxidation has been further described in the stressed right ventricle [27-29]. In terms of cellular mechanisms, we analysed BNP and $\beta$-MHC expression. Not surprisingly, BNP expression was inversely associated with RV contractile reserve measured by peak SVI. Combined with a significant increase in RVEDD and RVEDAI in PH animals, this result suggests that RV enlargement is strongly associated with impaired response to dobutamine in our large animal model. By contrast, we showed that $\beta$-MHC expression was higher in animals with low contractile reserve. $\beta$-MHC is mainly involved in weak contractions with slow shortening velocity, and may not play a major role in RV contractility in response to acute pharmacological stress [30]. We previously reported that $\beta$-MHC is upregulated after 12 weeks in our model of chronic RV pressure overload [14]. The present findings suggest that impaired RV contractile reserve is associated with increased $\beta$-MHC expression in the setting of chronic pressure overload.

\section{Clinical implications}

This study has two main clinical implications. First, low-dose infusion of dobutamine may be used in practice as a pharmacological stress to measure RV reserve. Secondly, the study highlights that contractile 
reserve could be more useful than resting parameters for risk stratification. In a recent study, BLUMBERG et al. [31] showed that CI at peak exercise was more strongly associated with outcome than resting parameters. In the present study, CI was increased in the PH group after dobutamine infusion, but to a lesser extent compared with controls. Our results suggest that indices of RV contractile reserve may be relevant markers of RV dysfunction in chronic pressure overload conditions. However, further studies are needed to document the incremental value of contractile reserve to measures of RV strain or RV remodelling, which will require a large sample size. The study also points to the fact that for the management of patients with severe RV failure and PAH, low dose dobutamine may not significantly increase stroke volume and the majority of the increase in CI will be through a chronotropic response. The lusitropic effects of dobutamine could also help improve systemic or renal congestion.

\section{Study limitations}

The study has several limitations. First, the pericardium was opened in the model, which may decrease the effects of ventricular interdependence [32]. In fact, the adverse effects of RV enlargement on left ventricular function have been demonstrated to be significantly lower when the pericardium is open. Decreased left ventricular ejection fraction in $\mathrm{PH}$ animals may, in part, explain moderate changes in SVI and CI after dobutamine infusion in this group compared with controls, even if SVI and CI were not different between the groups at rest. Secondly, we did not use cardiac magnetic resonance imaging for more accurate measurement of RV volumes and function. The strength of our study was the ability to perform echocardiography, conductance measurements and the pharmacological stress test in $\mathrm{PH}$ animals in $2 \mathrm{~h}$, allowing us to analyse the relationships between invasive and noninvasive indices of RV function. Thirdly, we did not report the value of the preload-recruitable stroke work, which would have helped to discriminate the load-dependency of RV contractile performance. Another limitation is related to the effects of halogenated anaesthesia on ventricular-arterial coupling efficiency. KerBAUL et al. [33] showed that isoflurane markedly impairs RV-PA coupling both by increasing RV afterload and by decreasing RV contractility. This limitation needs, however, to be balanced with the recognition that all animals (PH and sham groups) underwent the same anaesthetic protocol.

\section{Conclusion}

We report for the first time a strong relationship between ventricular-arterial coupling and RV contractile reserve in a porcine model of chronic pressure overload. This relationship offers a physiological basis to use contractile reserve as a surrogate marker of ventricular-arterial coupling in the chronic RV pressure overload state. Furthermore, the relationship explains the lower responsiveness to dobutamine in patients with severe baseline impairment in ventricular-arterial coupling.

\section{References}

1 Humbert M. Pulmonary arterial hypertension and chronic thromboembolic pulmonary hypertension: pathophysiology. Eur Respir Rev 2010; 19: 59-63.

2 Bogaard HJ, Abe K, Vonk Noordegraaf A, et al. The right ventricle under pressure: cellular and molecular mechanisms of right-heart failure in pulmonary hypertension. Chest 2009; 135: 794-804.

3 Vonk-Noordegraaf A, Haddad F, Chin KM, et al. Right heart adaptation to pulmonary arterial hypertension: physiology and pathobiology. J Am Coll Cardiol 2013; 62: Suppl. 25, D22-D33.

4 Humbert M, Sitbon O, Chaouat A, et al. Survival in patients with idiopathic, familial, and anorexigen-associated pulmonary arterial hypertension in the modern management era. Circulation 2010; 122: 156-163.

5 Haddad F, Doyle R, Murphy DJ, et al. Right ventricular function in cardiovascular disease, part II: pathophysiology, clinical importance, and management of right ventricular failure. Circulation 2008; 117: 1717-1731.

6 Haddad F, Vrtovec B, Ashley EA, et al. The concept of ventricular reserve in heart failure and pulmonary hypertension: an old metric that brings us one step closer in our quest for prediction. Curr Opin Cardiol 2011; 26: 123-131.

7 Grünig E, Tiede $\mathrm{H}$, Enyimayew EO, et al. Assessment and prognostic relevance of right ventricular contractile reserve in patients with severe pulmonary hypertension. Circulation 2013; 128: 2005-2015.

8 Nootens M, Wolfkiel CJ, Chomka EV, et al. Understanding right and left ventricular systolic function and interactions at rest and with exercise in primary pulmonary hypertension. Am J Cardiol 1995; 75: 374-377.

9 Brimioulle S, Wauthy P, Ewalenko P, et al. Single-beat estimation of right ventricular end-systolic pressure-volume relationship. Am J Physiol Heart Circ Physiol 2003; 284: H1625-H1630.

10 Vonk Noordegraaf A, Galie N. The role of the right ventricle in pulmonary arterial hypertension. Eur Respir Rev 2011; 20: 243-253.

11 Guihaire J, Haddad F, Boulate D, et al. Non-invasive indices of right ventricular function are markers of ventricular-arterial coupling rather than ventricular contractility: insights from a porcine model of chronic pressure overload. Eur Heart J Cardiovasc Imaging 2013; 14: 1140-1149.

12 Kelley KW, Curtis SE, Marzan GT, et al. Body surface area of female swine. J Anim Sci 1973; 36: 927-930.

13 Dickstein ML, Yano O, Spotnitz HM, et al. Assessment of right ventricular contractile state with the conductance catheter technique in the pig. Cardiovasc Res 1995; 29: 820-826.

14 Guihaire J, Haddad F, Boulate D, et al. Right ventricular plasticity in a porcine model of chronic pressure overload. J Heart Lung Transplant 2014; 33: 194-202. 
15 Kuehne T, Yilmaz S, Steendijk P, et al. Magnetic resonance imaging analysis of right ventricular pressure-volume loops: in vivo validation and clinical application in patients with pulmonary hypertension. Circulation 2004; 110: 2010-2016.

16 Kussmaul WG, Noordergraaf A, Laskey WK. Right ventricular-pulmonary arterial interactions. Ann Biomed Eng 1992; 20: 63-80.

17 Lambermont B, D'Orio V. The role of right ventricular-pulmonary arterial coupling to differentiate between effects of inotropic agents in experimental right heart failure. Crit Care Med 2006; 34: 2864-2865.

18 Morimont $\mathrm{P}$, Lambermont B, Ghuysen A, et al. Effective arterial elastance as an index of pulmonary vascular load. Am J Physiol Heart Circ Physiol 2008; 294: H2736-H2742.

19 Naeije R, Chesler N. Pulmonary circulation at exercise. Compr Physiol 2012; 2: 711-741.

20 Raina A, Vaidya A, Gertz ZM, et al. Marked changes in right ventricular contractile pattern after cardiothoracic surgery: implications for post-surgical assessment of right ventricular function. J Heart Lung Transplant 2013; 32: 777-783.

21 Fine NM, Chen L, Bastiansen PM, et al. Outcome prediction by quantitative right ventricular function assessment in 575 subjects evaluated for pulmonary hypertension. Circ Cardiovasc Imaging 2013; 6: 711-721.

22 Wong YY, Ruiter G, Lubberink M, et al. Right ventricular failure in idiopathic pulmonary arterial hypertension is associated with inefficient myocardial oxygen utilization. Circ Heart Fail 2011; 4: 700-706.

23 Wong YY, Westerhof N, Ruiter G, et al. Systolic pulmonary artery pressure and heart rate are main determinants of oxygen consumption in the right ventricular myocardium of patients with idiopathic pulmonary arterial hypertension. Eur J Heart Fail 2011; 13: 1290-1295.

24 Tomanek RJ. Response of the coronary vasculature to myocardial hypertrophy. J Am Coll Cardiol 1990; 15: 528-533.

25 Gómez A, Bialostozky D, Zajarias A, et al. Right ventricular ischemia in patients with primary pulmonary hypertension. J Am Coll Cardiol 2001; 38: 1137-1142.

26 Chin KM, Kim NH, Rubin LJ. The right ventricle in pulmonary hypertension. Coron Artery Dis 2005; 16: 13-18.

27 Wong YY, Raijmakers P, van Campen J, et al. ${ }^{11} \mathrm{C}$-acetate clearance as an index of oxygen consumption of the right myocardium in idiopathic pulmonary arterial hypertension: a validation study using ${ }^{15} \mathrm{O}$-labeled tracers and PET. J Nucl Med 2013; 54: 1258-1262.

28 Bodi V, Husser O, Sanchis J, et al. Contractile reserve and extent of transmural necrosis in the setting of myocardial stunning: comparison at cardiac MR imaging. Radiology 2010; 255: 755-763.

29 Mélon PG, de Landsheere CM, Degueldre C, et al. Relation between contractile reserve and positron emission tomographic patterns of perfusion and glucose utilization in chronic ischemic left ventricular dysfunction: implications for identification of myocardial viability. I Am Coll Cardiol 1997; 30: 1651-1659.

30 Lowes BD, Minobe W, Abraham WT, et al. Changes in gene expression in the intact human heart. Downregulation of alpha-myosin heavy chain in hypertrophied, failing ventricular myocardium. J Clin Invest 1997; 100: $2315-2324$

31 Blumberg FC, Arzt M, Lange T, et al. Impact of right ventricular reserve on exercise capacity and survival in patients with pulmonary hypertension. Eur J Heart Fail 2013; 15: 771-775.

32 Brookes $\mathrm{C}$, Ravn $\mathrm{H}$, White $\mathrm{P}$, et al. Acute right ventricular dilatation in response to ischemia significantly impairs left ventricular systolic performance. Circulation 1999; 100: 761-767.

33 Kerbaul F, Rondelet B, Motte S, et al. Isoflurane and desflurane impair right ventricular-pulmonary arterial coupling in dogs. Anesthesiology 2004; 101: 1357-1362. 